\title{
Animation Deformity in Breast Cancer Patients and Mental Health: A Review
}

\author{
Madeline O. Jansen, K. Brendan Butler, David A. Jansen* \\ Tulane University School of Medicine, New Orleans, Louisiana, USA
}

\section{Article Info}

\section{Article Notes}

Received: January 2, 2019

Accepted: February 13, 2019

\section{*Correspondence:}

Dr. David A. Jansen, Chief of Plastic Surgery, Tulane

University School of Medicine, New Orleans, Louisiana,

USA; Email: drjansen@jansenplasticsurgery.com.

(c) 2019 Jansen DA. This article is distributed under the terms of the Creative Commons Attribution 4.0 International License.

\section{Keywords}

Animation deformity

Breast cancer

Breast reconstruction

Mental health

Quality of life

Depression

\section{Abstract}

Animation Deformity (AD) is a common and serious complication in breast cancer patients undergoing reconstructive surgery involving subpectoral breast implant placement. AD is associated with an increased risk of poor mental health outcomes, including diminished quality of life, depression, suicidality, body image dissatisfaction, and body dysmorphic disorder. Breast cancer survivors, especially those who elect to undergo reconstructive surgery, are known to be a vulnerable population for the development of mental health disorders. Because effective management of AD can be beneficial to a patient's psychological wellbeing, this represents an important area of consideration for the surgical community. There are several methods for treating AD, which can involve repositioning the implant or inhibiting pectoralis muscle contraction. Such methods include manual muscle transection, neuromodulation, and selective nerve ablation. Research efforts have yet to examine which techniques produce more satisfactory improvements in psychosocial health. Thus, this may be an important area of focus for future research. This review examines contemporary research findings in order to broaden understandings of the relationship between $A D$ and mental health, as well to highlight the importance of managing this complication.

\section{Introduction}

Treatment of breast cancer often involves mastectomy, the removal of diseased breast tissue, followed by surgical reconstruction of the breast ${ }^{1}$. In recent years, breast reconstruction has become increasingly utilized as a component of breast cancer management ${ }^{2}$. In 2014, for example, $40 \%$ of breast cancer patients undergoing mastectomy also elected to have reconstructive surgeries ${ }^{3}$. Breast reconstruction can be an important aspect of the healing process, and is associated with positive psychosocial gains ${ }^{4}$. However, complications of this surgery have been shown to negatively impact mental health ${ }^{4}$. Animation deformity (AD) is a specific and distressing complication of breast reconstruction with subpectoral implant placement ${ }^{5}$. This can occur when breast implants are placed below the pectoralis muscle and contraction of the muscle leads to visible distortion of the breast tissue $^{6}$. Figures 1 and 2 demonstrate the characteristic implant displacement that often occurs during pectoralis muscle contraction in AD. Breast cancer patients appear to be especially sensitive to this complication, with reported incidence of up to $78 \%{ }^{7,8}$.

\section{Animation Deformity and Poor Mental Health Outcomes}

Studies have shown an association between $\mathrm{AD}$ and poor mental health outcomes such as decreased quality of life ${ }^{9}$ and depression ${ }^{10}$. Dimensions of quality of life strongly associated with $\mathrm{AD}$ include the perception of diminished emotional and psychological health, 


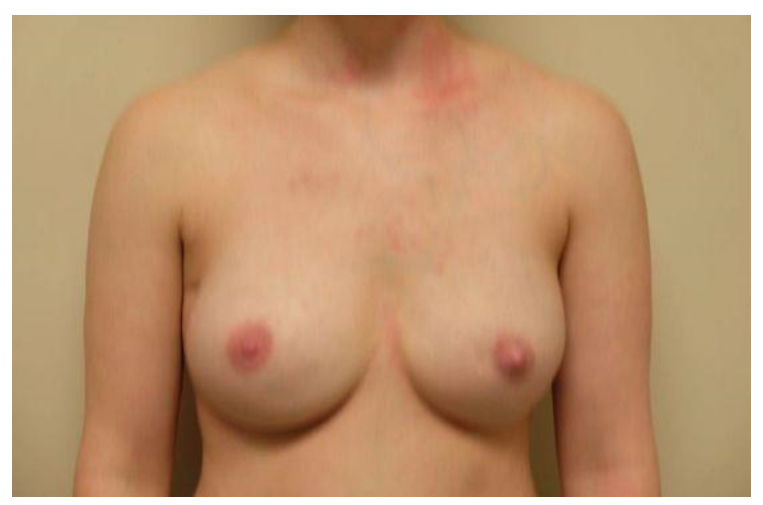

Figure 1: Pre-op, relaxed

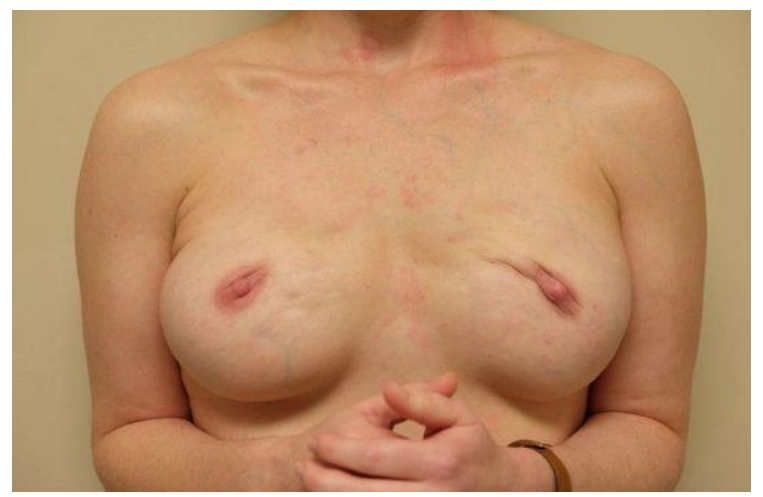

Figure 2: Pre-op, pectoral muscle activation

decreased ability to engage in activities of daily living, and difficulties with personal relationships ${ }^{9}$. The magnitude of this problem is illustrated by the finding that $80 \%$ of these patients report $\mathrm{AD}$ to be a bothersome complication that affects their everyday life ${ }^{9}$. Of additional significance are recent findings showing an association between breast asymmetry, a common feature of AD, an elevated risk of depression, and decreased psychosocial functioning ${ }^{10}$.

\section{Psychological Impact of Cosmetic Outcomes in Breast Reconstruction}

Breast cancer patients experiencing poor cosmetic results of breast reconstruction, such as $\mathrm{AD}$, are known to be at an increased risk of poor psychosocial outcomes ${ }^{11}$. For instance, a patient's negative perception of the cosmetic outcome has been shown to be detrimental to psychological health ${ }^{12}$. Higher levels of dissatisfaction are also associated with an increased risk of depression, anxiety, and disorders of body image ${ }^{13}$. Body image dissatisfaction is of clinical significance because it has been found to be related to lower quality of life in cancer patients ${ }^{14}$.

\section{Breast Cancer Patients Undergoing Reconstruction as a High Risk Population}

Breast cancer patients, especially those undergoing mastectomy and breast reconstruction, are known to be at an increased risk of psychiatric disorders ${ }^{15}$. Compared to the general population, major depressive disorder is seen at significantly higher rates among those with breast cancer ${ }^{16}$. In fact, the prevalence of depression in this group has been reported to be as high as $46 \%{ }^{17}$. Increased levels of hopelessness, a dimension of depression and risk factor for suicide, have also been observed among breast cancer patients receiving mastectomy ${ }^{18}$. Recent research also indicates an increased risk of suicide among those who have surgeries involving breast implants ${ }^{19}$. Other psychiatric comorbidities seen more frequently in breast cancer patients include posttraumatic stress disorder ${ }^{20}$ and generalized anxiety disorder ${ }^{21}$, with respective prevalences of $10 \%{ }^{20}$ and $16 \%{ }^{21}$.

Body image is another important factor to consider when discussing the psychological health of breast cancer survivors, with $50 \%{ }^{22}-75 \%{ }^{23}$ reporting significant levels of body image dissatisfaction. Those undergoing mastectomy and reconstruction, in particular, are known to experience the highest risk of negative body image ${ }^{18,22}$. Research also implicates a strong association between body image dissatisfaction, decreased mental health outcomes, and diminished self-esteem among breast cancer patients undergoing mastectomy and reconstruction ${ }^{22}$. Conversely, positive body image is associated with increased levels of self confidence in coping with cancer ${ }^{24}$. Moreover, body dysmorphic disorder (BDD), the disabling preoccupation with a minor or imagined defect, is significantly higher among breast cancer patients pursuing breast reconstruction following mastectomy ${ }^{25}$. This is significant because BDD has been shown to confer an elevated risk of depression and suicide ${ }^{26}$.

It should be noted that, compared to mastectomy alone, breast reconstruction following mastectomy has been shown to yield psychosocial benefits ${ }^{4}$. Although the underpinnings of this association have yet to be elucidated in the scientific literature, it may be related to body image and perception of cosmetic outcomes. As discussed above, these factors are known to impact psychosocial functioning in breast cancer survivors ${ }^{11,12,13,14}$.

\section{Implications for Treatment of Animation Deformity}

Addressing the psychological impact of breast cancer, subsequent breast reconstruction, and surgical complications, such as AD, is of great importance. Several studies have shown psychological factors to be associated with overall survival in these patients ${ }^{15}$. Therefore, it is essential to treat $\mathrm{AD}$, should it appear as a complication in breast cancer patients receiving mastectomy with subpectoral implant placement.

Implant-based techniques are the most common methods 
for breast reconstruction in patients who have undergone mastectomy ${ }^{27}$. Subpectoral, rather than pre-pectoral, placement of the implant is more commonly employed due to the belief that it yields a more natural appearing result ${ }^{28}$. It also has a decreased risk of capsular contracture, which is scarring around the implant ${ }^{28}$. Prevention of $\mathrm{AD}$ can be achieved through placement of the implant above the pectoralis muscle ${ }^{29}$. However, this is not a viable option for all patients, especially those receiving radiation therapy prior to surgery ${ }^{29}$. Options for treating AD include the following: repositioning the implant from subpectoral to prepectoral ${ }^{27}$; a muscle splitting biplanar technique, in which the implant is partially covered by the pectoralis muscle ${ }^{30}$; the injection of neuromodulators into the pectoralis muscle, which temporarily inhibits muscle contraction ${ }^{31}$; and complete transection of the pectoralis muscle ${ }^{32}$. Surgeries involving transection of the pectoralis muscle involve complete denervation of the tissue and subsequent muscular atrophy $^{33}$. An alternative method has been described in a recent case study that selectively denervated the pectoralis muscle through ablation of the medial and lateral pectoral nerves $^{5}$. Complete correction of $\mathrm{AD}$ was observed in this patient without long term muscle atrophy, indicating that this is another important consideration for this patient population $^{5}$ (see figures $1,2,3,4$ ).

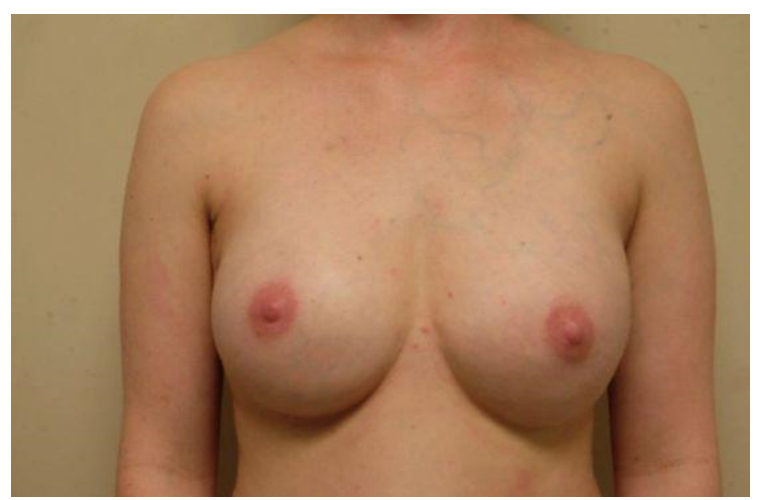

Figure 3: Post-op, selective nerve ablation technique, relaxed

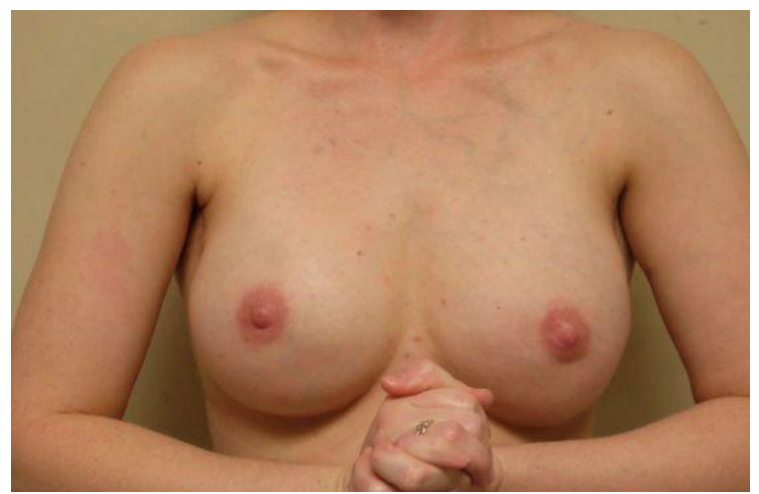

Figure 4: Post-op, selective nerve ablation technique, pectoral muscle activation

\section{Discussion}

While it is clear that treatment of $\mathrm{AD}$ is an important aspect of managing the psychosocial health of patients with breast cancer, it remains to be seen which surgical intervention yields the best mental health outcomes. Treatments that effectively correct the deformity and those that confer decreased risk of other complications will likely be the most successful. Because of the impact $\mathrm{AD}$ has on quality of life and psychosocial functioning, future research efforts should attempt to compare differences in mental health outcomes across the various AD treatment modalities.

Furthermore, because existent studies indicate there to be an association between AD and poor mental health outcomes, the nature of this relationship warrants future exploration. It may be necessary that studies be designed to evaluate more specifically how AD incurs an increased risk of depression and decreased quality of life. Further work may also explore potential associations between AD and other mental illnesses, such as anxiety disorders.

\section{Conclusion}

$\mathrm{AD}$ is a common and psychologically distressing complication of breast reconstruction among breast cancer survivors, who are at increased risk of poor mental health outcomes. Additionally, breast reconstruction and subsequent $\mathrm{AD}$ have been shown to be associated with a host of poor mental health outcomes. Examples include reduced quality of life, depression, body image dissatisfaction, and increased risk of suicide. Several options exist for the correction of $\mathrm{AD}$, but research has yet to examine which method confers the best psychological outcome.

\section{Acknowledgements}

There were no financial supports utilized in the writing of this review.

\section{Conflicts of Interest}

The authors declare that they have no competing interests.

\section{References}

1. Fox JP, Philip EJ, Gross CP, et al. Associations between mental health and surgical outcomes among women undergoing mastectomy for cancer. The breast journal. 2013 May; 19(3): 276-84.

2. Mays S, Alabdulkareem H, Christos P, et al. Surgical outcomes in women $\geq 70$ years undergoing mastectomy with and without reconstruction for breast cancer. The American Journal of Surgery. 2017 Nov 1; 214(5): 904-6.

3. Miller AM, Steiner CA, Barrett ML, et al. Breast Reconstruction Surgery for Mastectomy in Hospital Inpatient and Ambulatory Settings, 20092014: Statistical Brief\# 228.

4. Atisha D, Alderman AK, Lowery JC, et al. Prospective analysis of long-term psychosocial outcomes in breast reconstruction: twoyear postoperative results from the Michigan Breast Reconstruction Outcomes Study. Annals of surgery. 2008 Jun 1; 247(6): 1019-28. 
5. Eck DL, Nguyen, DC, Barnes, LL, et al. Treatment of Breast Animation Deformity in Implant-based Reconstruction with Selective Nerve Ablation: A Case Report. Aesthetic Plast Surg. 2018 Dec; 42(6): 14721475.

6. Pérez-San-Gregorio MÁ, Martín-Rodríguez A, Arias-Moreno MJ, et al. Self-reported psychological development in cosmetic breast surgery patients. Medicine. 2016 Dec 9; 95 (49): e5620.

7. Nigro LC, Blanchet NP. Animation Deformity in Postmastectomy Implant-Based Reconstruction. Plastic and Reconstructive Surgery Global Open. 2017 Jul; 5(7).

8. Spear SL, Schwartz J, Dayan JH, et al. Outcome assessment of breast distortion following submuscular breast augmentation. Aesthetic plastic surgery. 2009 Jan 1; 33(1): 44-8.

9. Becker H, Fregosi N. The impact of animation deformity on quality of life in post-mastectomy reconstruction patients. Aesthetic surgery journal. 2017 Feb 3; 37(5): 531-6.

10. Kim MK, Kim T, Moon HG, et al. Effect of cosmetic outcome on quality of life after breast cancer surgery. European Journal of Surgical Oncology (EJSO). 2015 Mar 1; 41(3): 426-32.

11. Eltahir Y, Werners LL, Dreise MM, et al. Quality-of-life outcomes between mastectomy alone and breast reconstruction: comparison of patient-reported BREAST-Q and other health-related quality-of-life measures. Plastic and reconstructive surgery. 2013 Aug 1; 132(2): 201e-9e.

12. Nicholson RM, Leinster S, Sassoon EM. A comparison of the cosmetic and psychological outcome of breast reconstruction, breast conserving surgery and mastectomy without reconstruction. The breast. 2007 Aug 1; 16(4): 396-410.

13. Al-Ghazal SK, Fallowfield L, Blamey RW. Does cosmetic outcome from treatment of primary breast cancer influence psychosocial morbidity. European Journal of Surgical Oncology. 1999 Dec 1; 25(6): 571-3.

14. Lehmann V, Hagedoorn M, Tuinman MA. Body image in cancer survivors: a systematic review of case-control studies. Journal of Cancer Survivorship. 2015 Jun 1; 9(2): 339-48.

15. Montazeri A. Health-related quality of life in breast cancer patients: a bibliographic review of the literature from 1974 to 2007 . Journal of experimental \& clinical cancer research. 2008 Aug 29; 27(1): 1.

16. Shapiro SL, Lopez AM, Schwartz GE, et al. Quality of life and breast cancer: relationship to psychosocial variables. Journal of clinical psychology. 2001 Apr; 57(4): 501-19.

17. Krebber AMH, Buffart LM, Kleijn G, et al. Prevalence of depression in cancer patients: a meta-analysis of diagnostic interviews and selfreport instruments. Psycho-Oncology. 2014; 23 (2): 121-130.

18. Heidari M, Ghodusi M. The relationship between body esteem and hope and mental health in breast cancer patients after mastectomy. Indian journal of palliative care. 2015 May; 21(2): 198.

19. Manoloudakis N, Labiris G, Karakitsou N, et al. Characteristics of women who have had cosmetic breast implants that could be associated with increased suicide risk: a systematic review, proposing a suicide prevention model. Archives of plastic surgery. 2015 Mar; 42(2): 131.

20. Hegel MT, Moore CP, Collins ED, et al. Distress, psychiatric syndromes, and impairment of function in women with newly diagnosed breast cancer. Cancer. 2006 Dec 15; 107(12): 2924-31.

21. Lueboonthavatchai P. Prevalence and psychosocial factors of anxiety and depression in breast cancer patients. Journal-Medical Association of Thailand. 2007 Oct 1; 90(10): 2164.

22. Fobair P, Stewart SL, Chang S, et al. Body image and sexual problems in young women with breast cancer. Psycho-Oncology: Journal of the Psychological, Social and Behavioral Dimensions of Cancer. 2006 Jul; 15(7): 579-94.

23. Ganz PA, Rowland JH, Desmond K, et al. Life after breast cancer: understanding women's health-related quality of life and sexual functioning. Journal of Clinical Oncology. 1998 Feb; 16(2): 501-14.

24. Pikler V, Winterowd C. Racial and body image differences in coping for women diagnosed with breast cancer. Health psychology. 2003 Nov; 22(6): 632 .

25. Metcalfe DB, Duggal CS, Gabriel A, et al. Prevalence of body dysmorphic disorder among patients seeking breast reconstruction. Aesthetic surgery journal. 2014 Jul 1; 34(5): 733-7.

26. Angelakis I, Gooding PA, Panagioti M. Suicidality in body dysmorphic disorder (BDD): A systematic review with meta-analysis. Clinical psychology review. 2016 Nov 1; 49: 55-66.

27. Hammond DC, Schmitt WP, O'Connor EA. Treatment of breast animation deformity in implant-based reconstruction with pocket change to the subcutaneous position. Plastic and Reconstructive Surgery. 2015; 135(6): 1540-1544.

28. Salibian AA, Frey JD, Karp NS. Strategies and considerations in selecting between subpectoral and prepectoral breast reconstruction. Gland Surgery. 2018 Aug 14.

29. Lee M. Discussion: Treatment of Breast Animation Deformity in Implant-Based Reconstruction with Selective Nerve Ablation. Aesthetic plastic surgery. 2018 Dec 1; 42(6): 1476-7.

30. Khan U. Dynamic breasts: a common complication following partial submuscular augmentation and its correction using the musclesplitting biplane technique. Aesthetic Plast Surg. 2009; 33(3): 353360.

31. Figus A, Mazzocchi M, Dessy LA, et al. Treatment of muscular contraction deformities with botulinum toxin type A after latissimus dorsi flap and sub-pectoral implant breast reconstruction. Journal of Plastic, Reconstructive \& Aesthetic Surgery. 2009 Jul 1; 62(7): 869-75.

32. Maxwell GP, Tornambe R. Management of mammary subpectoral implant distortion. Clin Plast Surg. 1988; 15(4): 601-611.

33. Hoffman GW, Elliott LF. The anatomy of the pectoral nerves and its significance to the general and plastic surgeon. Ann Surg. 1987; 205(5): 504-506. 